\title{
Impacto económico de los inhibidores en hemofilia tipo A pediátrica
}

Araceli López-Facundo, ${ }^{1}$ Cecilia Rodríguez-Castillejos, ${ }^{1}$ Yulia Romero, ${ }^{2}$ Juan Gay y Diddier Prada * ${ }^{1}$ Instituto de Seguridad Social del Estado de México y Municipios, Hospital Materno Infantil, Estado de México; 'Zeed Pharmaceutical Solutions, Operaciones Clínicas, Ciudad de México; ${ }^{3}$ Tecnología e Información para la Salud, Ciudad de México; ${ }^{4}$ Instituto Nacional de Cancerología, Ciudad de México. México

\section{Resumen}

Introducción: Pocos estudios han evaluado el impacto económico de los inhibidores en hemofilia tipo A en México, especialmente en población pediátrica. Objetivo: Determinar el impacto económico que conlleva el desarrollo de inhibidores en pacientes pediátricos con hemofilia tipo A. Método: Se evaluaron de forma retrospectiva los pacientes con hemofilia tipo $A$ atendidos en un servicio de hematología pediátrica entre diciembre de 2015 y noviembre de 2017, y se determinaron los costos directos e indirectos a partir de la presencia o ausencia de inhibidores. Resultados: El análisis de costos de la población estudiada $(n=24)$ mostró que el diagnóstico, seguimiento, profilaxis, tratamiento y hospitalización de estos pacientes tuvo un costo de \$6 883187.4 anuales por paciente, de los cuales más de $95 \%$ dependió del uso de factores hemostáticos. El costo anual por paciente en el grupo con inhibidores tuvo un costo de $\$ 5548765.0$, en comparación con $\$ 1334422.4$ del grupo sin inhibidores, 4.2 veces superior. Conclusiones: Se trata del primer estudio nacional que muestra que el desarrollo de inhibidores en pacientes pediátricos con hemofilia tipo A eleva más de cuatro veces la erogación económica derivada de esta enfermedad.

PALABRAS CLAVE: Impacto económico. Costo anual. Hemofilia tipo A. Inhibidores. México.

\section{Abstract}

Introduction: Few studies have assessed the economic impact of inhibitors in hemophilia A in Mexico, especially in the pediatric population. Objective: To determine the economic impact entailed by the development of inhibitors in pediatric patients with hemophilia A. Method: Patients with hemophilia A under the care of a pediatric hematology department between December 2015 and November 2017 were retrospectively assessed. Direct and indirect costs were determined based on the presence or absence of inhibitors. Results: The cost analysis of the study population $(n=24)$ showed that diagnosis, follow-up, prophylaxis, treatment and hospitalization of these patients had an annual cost of \$ 6883187.4 per patient, out of which more than $95 \%$ depended on the use of hemostatic factors. Annual cost per patient in the group with inhibitors was \$5 548765.0 in comparison with \$1334 422.4 in the group without inhibitors, 4.2 times higher. Conclusions: This is the first national study to show that the presence of inhibitors in pediatric patients with hemophilia $A$ increases the cost of the disease more than four times.

KEY WORDS: Economic impact. Annual cost. Hemophilia A. Inhibitors. Mexico.

Correspondencia:

*Diddier Prada

E-mail: dpradao@incan.edu.mx
Fecha de recepción: 10-09-2018

Fecha de aceptación: 17-05-2019

DOI: $10.24875 / G M M .19004674$
Gac Med Mex. 2019;155:369-376

Disponible en PubMed www.gacetamedicademexico.com 


\section{Introducción}

La hemofilia es una enfermedad congénita rara, caracterizada por anormalidades genéticas que provocan la pérdida del funcionamiento del factor VIII (hemofilia tipo A) o del factor IX (hemofilia tipo B). ${ }^{1}$ La prevalencia de la hemofilia tipo A se estima en aproximadamente un caso por cada 5000 a 7000 nacimientos de varones. ${ }^{2}$ Los pacientes con hemofilia tipo $A \circ B$, especialmente en la forma clínica severa, presentan repetidas hemorragias prolongadas, espontáneas o postraumáticas. ${ }^{3}$ La profilaxis, especialmente si se inicia de forma temprana en el transcurso de la vida, se ha asociado con mejores desenlaces articulares. ${ }^{4}$ Los pacientes con hemofilia tipo A pueden desarrollar anticuerpos que neutralizan el factor de coagulación VIII que se les administra. ${ }^{3}$ Actualmente, el costo del tratamiento es uno de los aspectos más importantes por considerar en esta enfermedad, ${ }^{5}$ sin embargo, en nuestro país son pocos los estudios que han llevado a cabo un análisis de costos. ${ }^{6}$

Esta investigación tuvo como objetivo determinar los costos directos e indirectos del manejo de la hemofilia tipo $A$, con énfasis en el desarrollo de inhibidores.

\section{Método}

Este estudio fue llevado a cabo de forma retrospectiva en el Hospital Materno Infantil del Instituto de Seguridad Social del Estado de México y Municipios, unidad Toluca, México. Se evaluaron todos los pacientes pediátricos con hemofilia tipo $A$ atendidos entre diciembre de 2015 y noviembre de $2017(n=24)$. Este estudio fue aprobado por el Comité de Ética Institucional del hospital. Se llevó a cabo un registro minucioso de las características sociodemográficas, clínicas y de eventos hemorrágicos de los pacientes, incluyendo el tipo de factor administrado, interconsultas requeridas, pruebas de laboratorio requeridas, estudios de imagen y la determinación de factores de coagulación, entre otros.

Para el análisis de costos directos (derivados de la atención de la enfermedad), se tomó como base el año 2016, tomando los costos institucionales de ese año y se llevó a cabo el cálculo en pesos mexicanos. Para los costos indirectos (que no dependen directamente de la enfermedad de base) se obtuvieron los promedios del ingreso por hora trabajada y de las horas trabajadas a la semana en la población ocupada durante 2016 para México, determinados por el Instituto Nacional de Estadística y Geografía, y se incluyeron los días perdidos por hospitalización y los días usados para la atención en las consultas requeridas durante el periodo de estudio por parte de los padres o tutores del paciente. ${ }^{7,8}$ Los datos fueron evaluados con el software $R^{\circledR}$ (R Project for Statistical Computing, CRAN, The Comprehensive R Archive Network, Viena).

\section{Resultados}

Las características heredofamiliares y clínicas de los pacientes, tanto en pacientes con y sin inhibidores, se muestran en la Tabla 1. Los detalles de los eventos hemorrágicos se muestran en la Tabla 2.

\section{Costos directos}

El costo por consultas médicas por paciente por año en el grupo sin inhibidores fue de $\$ 757.1$ (0.06\% del costo anual total por paciente), en comparación con $\$ 548.0$ en el grupo con inhibidores $(0.01 \%)$. En relación con las pruebas de laboratorio, el costo estimado por paciente por año para este rubro fue de $\$ 357.0$ en el subgrupo sin inhibidores $(0.03 \%$ del costo anual total por paciente) y de $\$ 131.8$ en el subgrupo con inhibidores $(0.01 \%)$. En relación con los estudios de imagen, el costo estimado para este rubro por paciente por año fue de $\$ 722.4$ (0.05\% del costo anual total por paciente) en el grupo sin inhibidores, en comparación con \$669.5 (0.05\%) en el grupo con inhibidores. El costo detallado de las consultas médicas, pruebas de laboratorio y de imagen registradas se muestran en la Tabla 3.

\section{CONSUMO DE FACTORES HEMOSTÁTICOS PARA PROFILAXIS Y EVENTOS HEMORRÁGICOS}

El costo anual por paciente del uso de factor VIII para profilaxis fue de $\$ 1141240.9$ (85.52 \% del costo anual total por paciente) en el grupo sin inhibidores y de $\$ 1783375.2$ (32.12\%) en el grupo con inhibidores. El costo anual de la profilaxis, incluyendo factores VII y VIII, fue de $\$ 36407522.3$, con un costo anual por paciente de \$1 141240.9 para el grupo sin inhibidores, en comparación con \$2 944788.9 en el grupo con inhibidores, lo que representa 2.6 veces más costo por profilaxis, asociado con la presencia de inhibidores. El costo del uso de factores hemostáticos para profilaxis detallado por paciente y durante todo el periodo de estudio se muestra en la Tabla 4. 
Tabla 1. Características heredofamiliares y clínicas de los pacientes pediátricos con hemofilia tipo A*

\begin{tabular}{|c|c|c|c|c|c|}
\hline \multirow{2}{*}{$\begin{array}{l}\text { Variables } \\
\text { Continuas }\end{array}$} & \multicolumn{2}{|c|}{ Sin inhibidores $(n=19)$} & \multicolumn{2}{|c|}{ Con inhibidores $(n=5)$} & \multirow[t]{2}{*}{ p } \\
\hline & Media/Mediana* & DE/RIC & Media/Mediana* & DE/RIC & \\
\hline Edad al diagnóstico (meses) & 16.00 & 28.00 & 9.50 & 7.50 & 0.346 \\
\hline Edad al momento del estudio (años) & 11.64 & 3.72 & 9.67 & 3.74 & 0.060 \\
\hline Factor VIII al diagnóstico (\%) & 0.03 & 0.02 & 0.03 & 0.03 & 0.996 \\
\hline Primer factor VIII registrado (\%) & 0.01 & 0.03 & 0.04 & 0.41 & 0.354 \\
\hline Número de familiares afectados & 3.00 & 3.50 & 2.00 & 0.00 & 0.717 \\
\hline Categóricas & $\mathrm{n}$ & $\%$ & $\mathrm{n}$ & $\%$ & \\
\hline $\begin{array}{l}\text { Sexo } \\
\text { Masculino }\end{array}$ & 19 & 100.0 & 5 & 100.0 & \\
\hline $\begin{array}{l}\text { Severidad al diagnóstico } \\
\text { Leve } \\
\text { Moderada } \\
\text { Severa }\end{array}$ & $\begin{array}{c}2 \\
10 \\
7\end{array}$ & $\begin{array}{l}10.5 \\
52.6 \\
36.8\end{array}$ & $\begin{array}{l}0 \\
4 \\
1\end{array}$ & $\begin{array}{c}0.0 \\
80.0 \\
20.0\end{array}$ & 0.504 \\
\hline $\begin{array}{l}\text { Recibió tratamiento a domicilio } \\
\text { Sí } \\
\text { No }\end{array}$ & $\begin{array}{c}9 \\
10\end{array}$ & $\begin{array}{l}47.4 \\
52.6\end{array}$ & $\begin{array}{l}2 \\
3\end{array}$ & $\begin{array}{l}40.0 \\
60.0\end{array}$ & 1.000 \\
\hline $\begin{array}{l}\text { Tratamiento en el domicilio** } \\
\text { Factor VII } \\
\text { Factor VIII } \\
\text { Sin dato** }\end{array}$ & $\begin{array}{c}0 \\
10 \\
9\end{array}$ & $\begin{array}{c}0.0 \\
100.0\end{array}$ & $\begin{array}{l}2 \\
1 \\
2\end{array}$ & $\begin{array}{l}66.7 \\
33.3\end{array}$ & 0.058 \\
\hline $\begin{array}{l}\text { Articulación diana (previa) } \\
\text { No } \\
\text { Sí } \\
\text { Sin dato** }\end{array}$ & $\begin{array}{l}14 \\
4 \\
1\end{array}$ & $\begin{array}{l}77.8 \\
22.2\end{array}$ & $\begin{array}{l}5 \\
0\end{array}$ & $\begin{array}{c}100.0 \\
0.0\end{array}$ & 0.622 \\
\hline
\end{tabular}

El costo anual por paciente por uso de factor VIII para la atención de eventos hemorrágicos fue de $\$ 187851.0$ ( $14.08 \%$ del costo anual total por paciente) en el grupo sin inhibidores y de $\$ 37422.0(0.67 \%)$ en el grupo con inhibidores. El costo del uso de factor VIII para la atención de eventos hemorrágicos, detallado por paciente, así como el costo durante todo el periodo de estudio y el costo anual individual se muestran en la Tabla 5. El costo anual por paciente por uso de factor VII para profilaxis fue de \$1 161413.7 (20.92\% del costo anual total por paciente) y para la atención de eventos hemorrágicos de \$2 560105.4 en el grupo con inhibidores (46.11\%). El factor VII no se administró a los pacientes sin inhibidores.

\section{AtENCIÓn InTRAHOSPITALARIA}

Los porcentajes de eventos de atención hospitalaria, motivo de hospitalización y necesidad de factor
VIII para cada uno de los grupos (sin inhibidores y con inhibidores) se muestran en la Tabla 6.

\section{Costos indirectos}

Se cuantificaron 260 días perdidos por la cohorte. El costo anual por paciente, por los días de ausentismo laboral en el grupo sin inhibidores fue de $\$ 1550.6$ (0.12\% del costo anual total por paciente), en comparación con $\$ 329.4$ (0.02\%) en el grupo con inhibidores.

\section{Costos totales}

El costo total estimado de la atención de los pacientes con hemofilia A durante el periodo de estudio fue de $\$ 106226$ 940.2, con un costo anual de \$53 113470.1 para los 24 pacientes incluidos, lo que representó un costo de $\$ 13766374.7$ por paciente 
Tabla 2. Eventos hemorrágicos en pacientes pediátricos con hemofilia tipo ${ }^{\ddagger}$

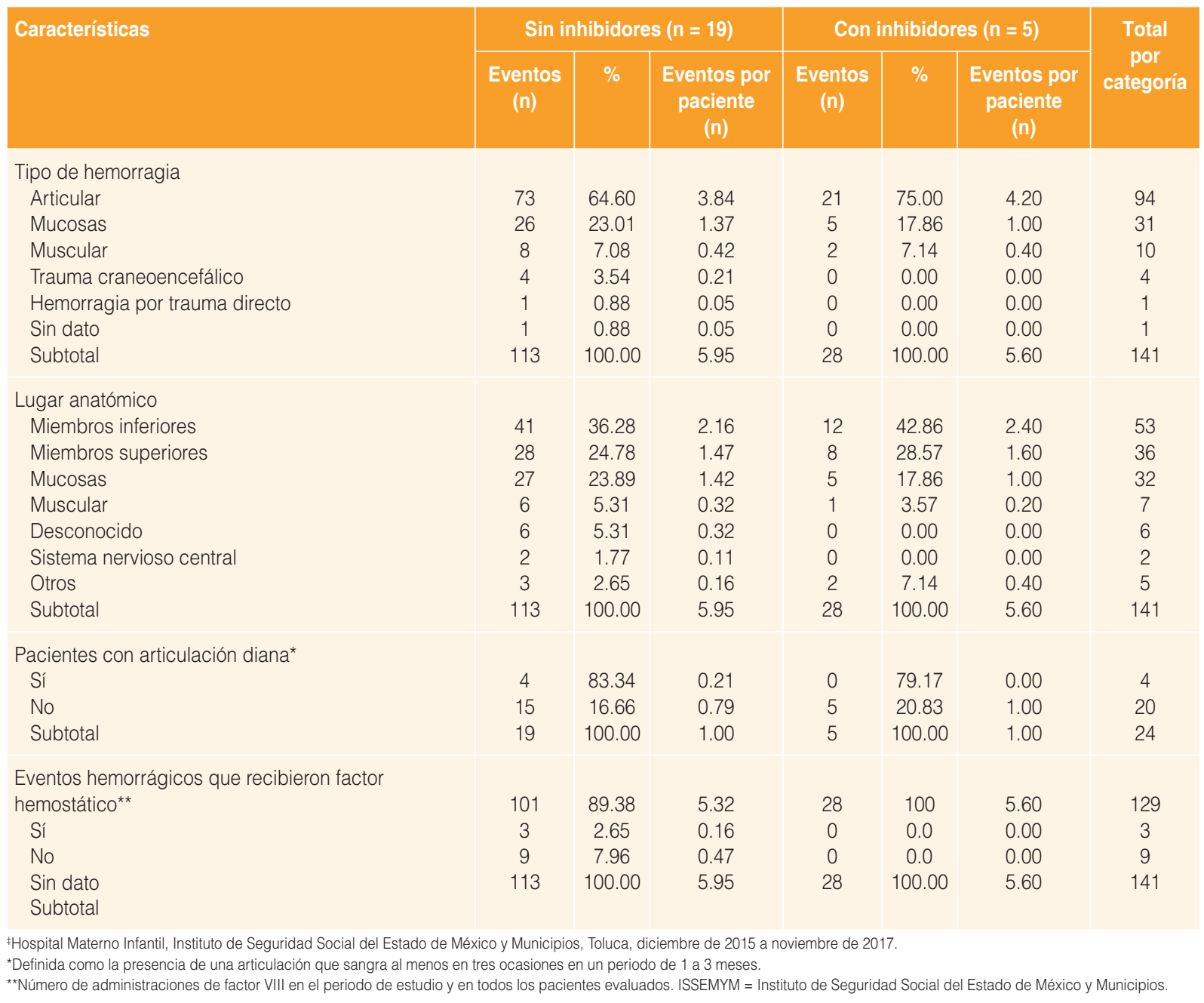

para todo el periodo de estudio y un costo anual promedio por paciente de $\$ 6883$ 187.4. El costo anual por paciente en el grupo sin inhibidores fue de \$1 334422.4 en comparación con \$5 548765.0 en el grupo con inhibidores, lo que representa un costo 4.2 veces mayor en el grupo con inhibidores. Como se observa en la Tabla 7, más de $95 \%$ del costo anual dependió del uso de factores de coagulación (factores VIII y VII), principalmente para profilaxis (\$61 200907.8 en todo el periodo de estudio para el factor VIII y \$11 614136.8 para el factor VII), pero también para el tratamiento de los eventos hemorrágicos y fue diferencial entre los subgrupos, con $99.6 \%$ para el uso de factor VIII en el subgrupo sin inhibidores, en comparación con $32.8 \%$ en el grupo con inhibidores. Sin embargo, el costo en el grupo con inhibidores por uso de factor VII correspondió a $67 \%$ del costo anual por paciente, el cual no fue requerido en el grupo sin inhibidores.

\section{Discusión}

En este estudio se encontró que la hemofilia tipo A presenta alta morbilidad, así como un impacto económico importante, el cual aumenta 4.2 veces en presencia de inhibidores. Se incluyeron todas las fuentes potenciales de costos en hemofilia tipo $\mathrm{A}$ y se describieron de forma detallada (consultas, estudios de laboratorio e imagen, atención de los eventos hemorrágicos, costos indirectos, etcétera) para un análisis holístico del impacto económico de la hemofilia (tipo $A$, dado que no se registraron casos de hemofilia tipo B) y de la presencia de inhibidores en población pediátrica.

Esta investigación es una continuación del trabajo académico que ha desarrollado nuestro grupo en relación con los costos de la hemofilia en México. ${ }^{9} \mathrm{El}$ análisis de costos mostró que más de $95 \%$ de los costos depende del uso de factores de coagulación, lo cual coincide con los hallazgos de múltiples publicaciones internacionales. ${ }^{8-10}$ Vale la pena resaltar que 
Tabla 3. Recursos consumidos por los pacientes pediátricos con hemofilia tipo A*

\begin{tabular}{|c|c|c|c|c|c|c|c|c|c|}
\hline \multirow[t]{2}{*}{ Variables } & \multicolumn{4}{|c|}{ Sin inhibidores $(n=19)$} & \multicolumn{4}{|c|}{ Con inhibidores $(n=5)$} & \multirow{2}{*}{$\begin{array}{c}\text { Total por } \\
\text { categoría } \\
\text { (\$ MXN) }\end{array}$} \\
\hline & $\begin{array}{c}\text { Eventos } \\
\text { (n) }\end{array}$ & $\begin{array}{c}\text { Costo } \\
\text { individual* } \\
\text { (MXN) }\end{array}$ & $\begin{array}{l}\text { Costo } \\
\text { total } \\
\text { en el } \\
\text { estudio** } \\
\text { (\$ MXN) }\end{array}$ & $\begin{array}{c}\text { Costo } \\
\text { anual } \\
\text { por } \\
\text { paciente } \\
\text { (\$ MXN) }\end{array}$ & $\begin{array}{c}\text { Eventos } \\
\text { (n) }\end{array}$ & $\begin{array}{c}\text { Costo } \\
\text { individual** } \\
\text { (\$ MXN) }\end{array}$ & $\begin{array}{c}\text { Costo } \\
\text { total en el } \\
\text { estudio** } \\
\text { (\$ MXN) }\end{array}$ & $\begin{array}{c}\text { Costo } \\
\text { anual } \\
\text { por } \\
\text { paciente } \\
\text { (\$ MXN) }\end{array}$ & \\
\hline Consultas médicas & 147 & 195.7 & 28767.9 & 757.1 & 28 & 195.7 & 5479.6 & 548.0 & 34247.5 \\
\hline $\begin{array}{l}\text { Estudios de laboratorio } \\
\text { Biometría hemática } \\
\text { Determinación del factor VIII de } \\
\text { coagulación } \\
\text { Tiempos de coagulación } \\
\text { Hepatitis B y C } \\
\text { Subtotal }\end{array}$ & $\begin{array}{c}6 \\
1 \\
18\end{array}$ & $\begin{array}{c}150.0 \\
1240.0 \\
\\
309.0 \\
1341.0 \\
3040.0\end{array}$ & $\begin{array}{c}450.0 \\
9920.0 \\
\\
1854.0 \\
1341.0 \\
13565.0\end{array}$ & $\begin{array}{c}11.8 \\
261.1 \\
\\
48.8 \\
35.3 \\
357.0\end{array}$ & $\begin{array}{l}1 \\
2 \\
2 \\
2 \\
1 \\
6\end{array}$ & $\begin{array}{c}150.0 \\
1240.0 \\
\\
309.0 \\
1341.0 \\
3040.0\end{array}$ & $\begin{array}{c}150.0 \\
2480.0 \\
\\
618.0 \\
1341.0 \\
4589.0\end{array}$ & $\begin{array}{c}15.0 \\
65.3 \\
\\
16.3 \\
35.3 \\
131.8\end{array}$ & $\begin{array}{c}600.0 \\
12400.0 \\
\\
2472.0 \\
2682.0 \\
18154.0\end{array}$ \\
\hline $\begin{array}{l}\text { Estudios de gabinete } \\
\text { Tomografía axial computarizada } \\
\text { Radiografía } \\
\text { Resonancia nuclear magnética } \\
\text { Ultrasonografía } \\
\text { Electroencefalograma } \\
\text { Subtotal }\end{array}$ & $\begin{array}{c}3 \\
10 \\
2 \\
1 \\
3 \\
19\end{array}$ & $\begin{array}{c}1937.0 \\
292.5 \\
7878.0 \\
708.0 \\
751.0 \\
11566.5\end{array}$ & $\begin{array}{c}5811.0 \\
2925.0 \\
15756.0 \\
708.0 \\
2253.0 \\
27453.0\end{array}$ & $\begin{array}{c}152.9 \\
77.0 \\
414.6 \\
18.6 \\
59.3 \\
722.4\end{array}$ & $\begin{array}{l}5 \\
0 \\
2 \\
0 \\
0 \\
7\end{array}$ & $\begin{array}{c}1937.0 \\
292.5 \\
7878.0 \\
708.0 \\
751.0 \\
11566.5\end{array}$ & $\begin{array}{c}9685.0 \\
0.0 \\
15756.0 \\
0.0 \\
0.0 \\
25441.0\end{array}$ & $\begin{array}{c}254.9 \\
0.0 \\
414.6 \\
0.0 \\
0.0 \\
669.5\end{array}$ & $\begin{array}{c}15496.0 \\
2925.0 \\
31512.0 \\
708.0 \\
2253.0 \\
52894.0\end{array}$ \\
\hline Subtotal & & & 83350.9 & 2193.4 & & & 40098.6 & 1055.2 & 123449.5 \\
\hline
\end{tabular}

${ }^{\star}$ Costo de cada evento (consulta, prueba de laboratorio, etcétera). Hospital Materno Infantil, Instituto de Seguridad Social del Estado de México y Municipios, Toluca, diciembre de 2015 a noviembre de 2017

**Incluye los costos de todos los eventos durante todo el periodo de estudio.

$\$ M X N=$ pesos mexicanos

Tabla 4. Días recibidos y unidades administradas de factor VIII para profilaxis en pacientes pediátricos con hemofilia tipo $A(n=24)^{*}$

\begin{tabular}{|c|c|c|c|c|c|c|}
\hline Paciente & $\begin{array}{l}\text { Días } \\
\text { recibidos } \\
\text { (n) }\end{array}$ & $\begin{array}{c}\text { Ul promedio } \\
\text { diarias recibidas }\end{array}$ & $\begin{array}{l}\text { Ul totales recibidas } \\
\text { durante el estudio }\end{array}$ & $\begin{array}{l}\text { Costo por } \\
\text { unidad } \\
\text { (\% MXN) }\end{array}$ & $\begin{array}{l}\text { Costo por } \\
\text { paciente } \\
\text { (\$ MXN) }\end{array}$ & $\begin{array}{l}\text { Costo anual } \\
\text { individual } \\
\text { (\$ MXN) }\end{array}$ \\
\hline $\begin{array}{c}\text { Sin inhibidores } \\
1 \\
2 \\
3 \\
4 \\
5 \\
6 \\
7 \\
8 \\
9 \\
10 \\
11 \\
12 \\
13 \\
14 \\
15 \\
\text { Subtotal }\end{array}$ & $\begin{array}{c}719 \\
547 \\
482 \\
377 \\
598 \\
633 \\
148 \\
612 \\
644 \\
552 \\
572 \\
564 \\
489 \\
21 \\
279 \\
7237\end{array}$ & $\begin{array}{c}591.7 \\
1163.5 \\
455.4 \\
631.3 \\
167.0 \\
250.0 \\
143.0 \\
625.0 \\
192.9 \\
476.6 \\
189.6 \\
250.0 \\
461.3 \\
750.0 \\
250.0 \\
6597.2\end{array}$ & $\begin{array}{c}425408.3 \\
636413.5 \\
219482.1 \\
237981.3 \\
99866.0 \\
158250.0 \\
21164.0 \\
382500.0 \\
124251.8 \\
263062.5 \\
108472.0 \\
141000.0 \\
225592.0 \\
15750.0 \\
69750.0 \\
3128943.4\end{array}$ & $\begin{array}{l}13.9 \\
13.9 \\
13.9 \\
13.9 \\
13.9 \\
13.9 \\
13.9 \\
13.9 \\
13.9 \\
13.9 \\
13.9 \\
13.9 \\
13.9 \\
13.9 \\
13.9\end{array}$ & $\begin{array}{c}5896159.5 \\
8820690.6 \\
3042022.5 \\
3298420.1 \\
1384142.8 \\
2193345.0 \\
293333.0 \\
5301450.0 \\
1722129.3 \\
3646046.3 \\
1503421.9 \\
1954260.0 \\
3126705.1 \\
218295.0 \\
966735.0 \\
43367156.0\end{array}$ & $\begin{array}{c}2948079.8 \\
4410345.3 \\
1521011.3 \\
1649210.1 \\
692071.4 \\
1096672.5 \\
146666.5 \\
2650725.0 \\
861064.6 \\
1823023.1 \\
751711.0 \\
977130.0 \\
1563352.6 \\
109147.5 \\
483367.5\end{array}$ \\
\hline $\begin{array}{l}\text { Con inhibidores } \\
1 \\
2 \\
3 \\
4 \\
5 \\
\text { Subtotal }\end{array}$ & $\begin{array}{c}238 \\
313 \\
628 \\
429 \\
207 \\
1815\end{array}$ & $\begin{array}{c}600.0 \\
1250.0 \\
690.8 \\
462.6 \\
581.6 \\
3585.0\end{array}$ & $\begin{array}{c}142800.0 \\
391250.0 \\
433815.8 \\
198455.4 \\
120385.3 \\
1286706.5\end{array}$ & $\begin{array}{l}13.9 \\
13.9 \\
13.9 \\
13.9 \\
13.9\end{array}$ & $\begin{array}{c}1979208.0 \\
5422725.0 \\
6012686.8 \\
2750591.8 \\
1668540.1 \\
17833751.7\end{array}$ & $\begin{array}{c}989604.0 \\
2711362.5 \\
3006343.4 \\
1375295.9 \\
834270.0\end{array}$ \\
\hline
\end{tabular}

*Hospital Materno Infantil, Instituto de Seguridad Social del Estado de México y Municipios, Toluca, diciembre de 2015 a noviembre de 2017.

$\mathrm{UI}=$ unidades internacionales, $\$ \mathrm{MXN}=$ pesos mexicanos. 
Tabla 5. Días recibidos y unidades administradas de factor VIII para la atención de eventos hemorrágicos en pacientes pediátricos con hemofilia tipo $A(n=24)^{*}$

\begin{tabular}{|c|c|c|c|c|c|c|}
\hline Paciente & Días recibidos (n) & $\begin{array}{l}\text { Ul promedio } \\
\text { diarias recibidas }\end{array}$ & $\begin{array}{l}\text { UI totales recibidas } \\
\text { durante el estudio }\end{array}$ & $\begin{array}{c}\text { Costo por } \\
\text { unidad } \\
\text { (\$ MXN) }\end{array}$ & $\begin{array}{l}\text { Costo por } \\
\text { paciente } \\
\text { (\$ MXN) }\end{array}$ & $\begin{array}{l}\text { Costo anual } \\
\text { individual } \\
\text { (\$ MXN) }\end{array}$ \\
\hline $\begin{array}{c}\text { Sin inhibidores } \\
1 \\
2 \\
3 \\
4 \\
5 \\
6 \\
7 \\
8 \\
9 \\
10 \\
11 \\
12 \\
13 \\
14 \\
15 \\
16 \\
17 \\
18 \\
19 \\
\text { Subtotal }\end{array}$ & $\begin{array}{c}3 \\
6 \\
6 \\
3 \\
76 \\
1 \\
3 \\
32 \\
5 \\
21 \\
3 \\
2 \\
47 \\
26 \\
7 \\
39 \\
26 \\
24 \\
222 \\
552\end{array}$ & $\begin{array}{c}608.3 \\
2333.3 \\
500.0 \\
916.7 \\
2220.5 \\
500.0 \\
750.0 \\
1103.9 \\
1450.0 \\
500.0 \\
625.0 \\
500.0 \\
583.3 \\
571.4 \\
2000.0 \\
921.9 \\
937.5 \\
1291.7 \\
533.3 \\
18846.8\end{array}$ & $\begin{array}{c}1825.0 \\
14000.0 \\
3000.0 \\
2750.0 \\
168754.5 \\
500.0 \\
2250.0 \\
35325.1 \\
7250.0 \\
10500.0 \\
1875.0 \\
1000.0 \\
27416.7 \\
14857.1 \\
14000.0 \\
35953.1 \\
24375.0 \\
31000.0 \\
118400.0 \\
515031.6\end{array}$ & $\begin{array}{l}13.9 \\
13.9 \\
13.9 \\
13.9 \\
13.9 \\
13.9 \\
13.9 \\
13.9 \\
13.9 \\
13.9 \\
13.9 \\
13.9 \\
13.9 \\
13.9 \\
13.9 \\
13.9 \\
13.9 \\
13.9 \\
13.9\end{array}$ & $\begin{array}{c}25294.5 \\
194040.0 \\
41580.0 \\
38115.0 \\
2338938.0 \\
6930.0 \\
31185.0 \\
489605.8 \\
100485.0 \\
145530.0 \\
25987.5 \\
13860.0 \\
379995.0 \\
205920.0 \\
194040.0 \\
498310.3 \\
337837.5 \\
429660.0 \\
1641024.0 \\
7138337.6\end{array}$ & $\begin{array}{c}12647.3 \\
97020.0 \\
20790.0 \\
19057.5 \\
1169469.0 \\
3465.0 \\
15592.5 \\
244802.9 \\
50242.5 \\
72765.0 \\
12993.8 \\
6930.0 \\
189997.5 \\
102960.0 \\
97020.0 \\
249155.2 \\
168918.8 \\
214830.0 \\
820512.0\end{array}$ \\
\hline $\begin{array}{l}\text { Con inhibidores } \\
1 \\
2 \\
3 \\
\text { Subtotal }\end{array}$ & $\begin{array}{c}2 \\
2 \\
31 \\
35\end{array}$ & $\begin{array}{c}750.0 \\
5000.0 \\
500.0 \\
6250.0\end{array}$ & $\begin{array}{c}1500.0 \\
10000.0 \\
15500.0 \\
27000.0\end{array}$ & $\begin{array}{l}13.9 \\
13.9 \\
13.9\end{array}$ & $\begin{array}{c}20790.0 \\
138600.0 \\
214830.0 \\
374220.0\end{array}$ & $\begin{array}{c}10395.0 \\
69300.0 \\
107415.0\end{array}$ \\
\hline
\end{tabular}

*Hospital Materno Infantil, Instituto de Seguridad Social del Estado de México y Municipios, Toluca, diciembre de 2015 a noviembre de 2017. $\mathrm{UI}=$ unidades internacionales, $\$ \mathrm{MXN}=$ peso mexicanos.

Tabla 6. Eventos de atención hospitalaria en pacientes pediátricos con hemofilia tipo A con y sin inhibidores $(n=24)^{*}$

\begin{tabular}{|c|c|c|c|c|c|c|}
\hline \multirow[t]{2}{*}{ Eventos } & \multirow{2}{*}{$\begin{array}{c}\text { Total } \\
\text { (n) }\end{array}$} & \multicolumn{2}{|c|}{ Sin inhibidores $(n=19)$} & \multicolumn{2}{|c|}{ Con inhibidores $(n=5)$} & \multirow[t]{2}{*}{ p } \\
\hline & & $\begin{array}{c}\text { Eventos } \\
\text { (n) }\end{array}$ & $\% * *$ & $\begin{array}{c}\text { Eventos } \\
\text { (n) }\end{array}$ & $\%^{* *}$ & \\
\hline Eventos de atención hospitalaria & 59 & 46 & 100.00 & 13 & 100.00 & \\
\hline $\begin{array}{l}\text { Consulta al servicio de urgencias } \\
\text { Atención en sala de urgencias sin hospitalización } \\
\text { Atención en sala de urgencias con hospitalización } \\
\text { Hospitalización por hematología }\end{array}$ & $\begin{array}{c}49 \\
33 \\
9 \\
7\end{array}$ & $\begin{array}{l}37 \\
28 \\
4 \\
5\end{array}$ & $\begin{array}{l}75.68 \\
10.81 \\
13.51\end{array}$ & $\begin{array}{l}12 \\
5 \\
5 \\
2\end{array}$ & $\begin{array}{l}41.67 \\
41.67 \\
16.67\end{array}$ & 0.043 \\
\hline $\begin{array}{l}\text { Motivo de hospitalización } \\
\text { Profilaxis } \\
\text { Hemorragia } \\
\text { Infección } \\
\text { Trauma } \\
\text { Cirugía } \\
\text { Otros } \\
\text { Desconocido }\end{array}$ & $\begin{array}{c}22 \\
10 \\
5 \\
2 \\
1 \\
8 \\
11\end{array}$ & $\begin{array}{c}22 \\
1 \\
5 \\
2 \\
1 \\
5 \\
10\end{array}$ & $\begin{array}{c}47.83 \\
2.17 \\
10.87 \\
4.35 \\
2.17 \\
10.87 \\
21.74\end{array}$ & $\begin{array}{l}0 \\
9 \\
1 \\
0 \\
0 \\
2 \\
1\end{array}$ & $\begin{array}{c}0.00 \\
69.23 \\
7.69 \\
0.00 \\
0.00 \\
15.38 \\
7.69\end{array}$ & $<0.001$ \\
\hline $\begin{array}{l}\text { Necesidad de factor VIII durante la hospitalización } \\
\text { Sí } \\
\text { No } \\
\text { Desconocido }\end{array}$ & $\begin{array}{l}35 \\
13 \\
11\end{array}$ & $\begin{array}{c}30 \\
6 \\
10\end{array}$ & $\begin{array}{l}83.33 \\
16.67\end{array}$ & $\begin{array}{l}5 \\
7 \\
1\end{array}$ & $\begin{array}{l}41.67 \\
58.33\end{array}$ & 0.0148 \\
\hline
\end{tabular}

*Hospital Materno Infantil, Instituto de Seguridad Social del Estado de México y Municipios, Toluca, diciembre de 2015 a noviembre de 2017.

**En relación con el total de los eventos. 
Tabla 7. Costo de la atención de pacientes pediátricos con hemofilia tipo A*

\begin{tabular}{|c|c|c|c|c|c|}
\hline & $\begin{array}{l}\text { Costo total en el } \\
\text { periodo de estudio } \\
\text { (\$ MXN) }\end{array}$ & $\begin{array}{c}\text { Costo por paciente en el } \\
\text { periodo de estudio } \\
\text { (\$ MXN) }\end{array}$ & $\begin{array}{c}\text { Costo anual } \\
(\$ \mathrm{MXN})\end{array}$ & $\begin{array}{c}\text { Costo anual por } \\
\text { paciente } \\
\text { (\$ MXN) }\end{array}$ & $\% * *$ \\
\hline $\begin{array}{l}\text { Sin inhibidores }(\mathrm{n}=19) \\
\text { Consultas } \\
\text { Estudios de laboratorio } \\
\text { Estudios de gabinete }\end{array}$ & $\begin{array}{l}28767.9 \\
13565.0 \\
27453.0\end{array}$ & $\begin{array}{l}1514.1 \\
713.9 \\
1444.9\end{array}$ & $\begin{array}{c}14384.0 \\
6782.5 \\
13726.5\end{array}$ & $\begin{array}{l}757.1 \\
357.0 \\
722.4\end{array}$ & $\begin{array}{l}0.06 \\
0.03 \\
0.05\end{array}$ \\
\hline $\begin{array}{l}\text { Factor VIII } \\
\text { Profilaxis } \\
\text { Eventos hemorrágicos }\end{array}$ & $\begin{array}{c}43367156.0 \\
7138337.6\end{array}$ & $\begin{array}{c}2282481.9 \\
375702.0\end{array}$ & $\begin{array}{c}21683578.0 \\
3569168.8\end{array}$ & $\begin{array}{c}1141240.9 \\
187851.0\end{array}$ & $\begin{array}{l}85.52 \\
14.08\end{array}$ \\
\hline $\begin{array}{l}\text { Factor VII } \\
\text { Profilaxis } \\
\text { Eventos hemorrágicos }\end{array}$ & $\begin{array}{l}0.0 \\
0.0\end{array}$ & $\begin{array}{l}0.0 \\
0.0\end{array}$ & $\begin{array}{l}0.0 \\
0.0\end{array}$ & $\begin{array}{l}0.0 \\
0.0\end{array}$ & $\begin{array}{l}0.00 \\
0.00\end{array}$ \\
\hline Consulta al servicio de urgencias & 14911.0 & 784.8 & 7455.5 & 392.4 & 0.03 \\
\hline Hospitalización & 58936.8 & 3101.9 & 29468.4 & 1551.0 & 0.12 \\
\hline Costos indirectos & 58923.0 & 3101.2 & 29461.5 & 1550.6 & 0.12 \\
\hline Total & 50708050.3 & 2668844.8 & 25354025.2 & 1334422.4 & 100.00 \\
\hline $\begin{array}{l}\text { Con inhibidores }(n=5) \\
\text { Consultas } \\
\text { Estudios de laboratorio } \\
\text { Estudios de gabinete }\end{array}$ & $\begin{array}{c}5479.6 \\
4589.0 \\
25441.0\end{array}$ & $\begin{array}{c}1095.9 \\
263.6 \\
1339.0\end{array}$ & $\begin{array}{c}2739.8 \\
2294.5 \\
12720.5\end{array}$ & $\begin{array}{l}548.0 \\
131.8 \\
669.5\end{array}$ & $\begin{array}{l}0.01 \\
0.01 \\
0.05\end{array}$ \\
\hline $\begin{array}{l}\text { Factor VIII } \\
\text { Profilaxis } \\
\text { Eventos hemorrágicos }\end{array}$ & $\begin{array}{c}17833751.7 \\
374220.0\end{array}$ & $\begin{array}{c}3566750.3 \\
74844.0\end{array}$ & $\begin{array}{c}8916875.9 \\
187110.0\end{array}$ & $\begin{array}{c}1783375.2 \\
37422.0\end{array}$ & $\begin{array}{c}32.12 \\
0.67\end{array}$ \\
\hline $\begin{array}{l}\text { Factor VII } \\
\text { Profilaxis } \\
\text { Eventos hemorrágicos }\end{array}$ & $\begin{array}{l}11614136.8 \\
25601054.3\end{array}$ & $\begin{array}{l}2322827.4 \\
5120210.9\end{array}$ & $\begin{array}{c}5807068.4 \\
12800527.2\end{array}$ & $\begin{array}{l}1161413.7 \\
2560105.4\end{array}$ & $\begin{array}{l}20.92 \\
46.11\end{array}$ \\
\hline Consulta al servicio de urgencias & 4836.0 & 967.2 & 2418.0 & 483.6 & 0.01 \\
\hline Hospitalización & 42864.1 & 8572.8 & 21432.0 & 4286.4 & 0.08 \\
\hline Costos indirectos & 12517.3 & 658.8 & 6258.7 & 329.4 & 0.02 \\
\hline Total & 55518889.9 & 11097530.0 & 27759444.9 & 5548765.0 & 100.00 \\
\hline
\end{tabular}

*Hospital Materno Infantil, Instituto de Seguridad Social del Estado de México y Municipios, Toluca, diciembre de 2015 a noviembre de 2017.

**Porcentaje del costo anual por paciente. \$MXN = pesos mexicanos.

la mayoría de los pacientes (> $90 \%$ ) recibió factores hemostáticos para el tratamiento de la enfermedad y un buen porcentaje de estos, tratamiento profiláctico. Esto sugiere que en México, a pesar de las limitaciones económicas, se está procurando llevar a cabo esquemas similares a los recomendados por la World Federation of Hemophilia y las Guías Mexicanas de Práctica Clínica para el Diagnóstico y Tratamiento de la Hemofilia en Población Pediátrica. ${ }^{11,12}$ Estas intervenciones tienen un impacto sustancial en relación con la disminución en la frecuencia de eventos hemorrágicos y en la morbilidad asociada, así como en la discapacidad a largo plazo.13,14 Este estudio también mostró elevada frecuencia de hemorragias en las mucosas, lo cual pudiera estar relacionado con un bajo reporte de este tipo de sangrados, dado su bajo impacto sobre la morbilidad de los pacientes.

En virtud de los elevados costos del tratamiento con factores de coagulación, los costos indirectos parecieran ser bajos ( $0.02 \%$ del costo total), sin embargo, esto puede tener grandes implicaciones en las familias de los pacientes afectados. El presente estudio explora todas las fuentes potenciales de costos dentro del proceso de atención de pacientes con hemofilia a partir de la revisión de los expedientes particulares. Otros análisis han tratado de recrear el panorama del impacto e impacto económico a partir de cuantificaciones más subjetivas (encuestas a especialistas), ${ }_{11}^{11}$ pero que contribuyen a un mejor entendimiento del impacto nacional de esta enfermedad. 
El presente estudio tiene importantes limitaciones, por ejemplo, corresponde a pacientes atendidos en una sola institución; a pesar de ello, aporta información valiosa dado que la hemofilia sigue siendo una enfermedad poco frecuente, además de caracterizar de forma detallada a 24 casos pediátricos, de los cuales aproximadamente $20 \%$ desarrolló inhibidores. El bajo número de casos también limitó los abordajes estadísticos multivariables, los cuales son fundamentales para determinar asociaciones independientes, por lo que las conclusiones clínicas siguen siendo limitadas.

Nuestra investigación demuestra, bajo la perspectiva de una institución pública nacional, que el desarrollo de inhibidores en población pediátrica con hemofilia tipo A aumenta sustancialmente los costos de la enfermedad.

\section{Agradecimientos}

Los autores agradecen a Novo Nordisk México S.A. de C.V., el apoyo financiero necesario para la realización del presente estudio y al respecto declaran que no tienen ningún interés competente, más allá que el haber recibido el patrocinio. El diseño del estudio, levantamiento y análisis de datos, así como la redacción del presente documento y su sometimiento para publicación se llevaron a cabo bajo absoluta autonomía.

\section{Bibliografía}

1. Gringeri A, Lundin B, von Mackensen S, Mantovani L, Mannucci PM, ESPRIT Study Group. A randomized clinical trial of prophylaxis in children with hemophilia A (the ESPRIT Study). J Thromb Haemost. 2011;9:700-710.

2. Qu Y, Nie X, Yang Z, Yin H, Pang Y, Dong P, et al. The prevalence of hemophilia in mainland China: a systematic review and meta-analysis. Southeast Asian J Trop Med Public Health. 2014;45:455-466.

3. Valentino LA, Hakobyan N, Enockson C, Simpson ML, Kakodkar NC, Cong $\mathrm{L}$, et al. Exploring the biological basis of haemophilic joint disease: experimental studies. Haemophilia. 2012;18:310-318.

4. Ljung R, Gretenkort-Andersson N. The current status of prophylactic replacement therapy in children and adults with haemophilia. $\mathrm{Br} \mathrm{J}$ Haematol. 2015;169:777-786.

5. Rocino A, Cortesi PA, Scalone L, Mantovani LG, Crea R, Gringeri A, et al. Immune tolerance induction in patients with haemophilia $a$ and inhibitors: effectiveness and cost analysis in an European Cohort (The ITER Study). Haemophilia. 2016;22:96-102.

6. Carlos-Rivera F, Gasca-Pineda R, Majluf-Cruz A, García-Chávez J. Economic impact of hemophilia type A and B in Mexico. Gac Med Mex. 2016;152:19-29.

7. Zhou ZY, Koerper MA, Johnson KA, Riske B, Baker JR, Ullman M, et al. Burden of illness: direct and indirect costs among persons with hemophilia A in the United States. J Med Econ. 2015;18:457-65.

8. Shrestha A, Eldar-Lissai A, Hou N, Lakdawalla DN, Batt K. Real-world resource use and costs of haemophilia A-related bleeding. Haemophilia. 2017;23(4):e267-e275.

9. Castillón-Benavides NK. Impacto económico del tratamiento de pacientes pediátricos con hemofilia tipo A en el Hospital Materno Infantil de ISSEMyM [tesis de posgrado]. México: Universidad Autónoma del Estado de México; 2016.

10. Gringeri A, Mantovani LG, Scalone L, Mannucci PM, COCIS Study Group. Cost of care and quality of life for patients with hemophilia complicated by inhibitors: the COCIS Study Group. Blood. 2003;102:2358-2363.

11. Srivastava A, Brewer AK, Mauser-Bunschoten EP, Key NS, Kitchen S, Llinas $A$, et al. Guidelines for the management of hemophilia. Haemophilia. 2013;19:e1-e47

12. Secretaría de Salud. Guía de práctica clínica. Diagnóstico y tratamiento de hemofilia en adultos. México: Secretaría de Salud; 2010.

13. Fernandes S, Carvalho M, Lopes M, Araújo F. Impact of an individualized prophylaxis approach on young adults with severe hemophilia. Semin Thromb Hemost. 2014;40:785-789.

14. Balkaransingh $P$, Young $G$. Novel therapies and current clinical progress in hemophilia A. Ther Adv Hematol. 2018;9:49-61. 